\title{
Aging influence on gray matter structural associations within the default mode network utilizing Bayesian network modeling
}

\author{
Yan Wang ${ }^{1}$, Kewei Chen ${ }^{2}$, Jiacai Zhang ${ }^{1}$, Li Yao ${ }^{1,3}$, Ke Li ${ }^{4}$, Zhen Jin ${ }^{4}$, Qing Ye ${ }^{1}$ and Xiaojuan Guo ${ }^{1,3}$ * \\ College of Information Science and Technology, Beijing Normal University, Beijing, China \\ ${ }^{2}$ Banner Alzheimer's Institute and Banner Good Samaritan PET Center, Phoenix, AZ, USA \\ ${ }^{3}$ State Key Laboratory of Cognitive Neuroscience and Learning, Beijing Normal University, Beijing, China \\ ${ }^{4}$ Laboratory of Magnetic Resonance Imaging, The 306th Hospital of People's Liberation Army, Beijing, China
}

\section{Edited by:}

Rodrigo Orlando Kuljiš, Zdrav Mozak

Limitada, Chile

Reviewed by:

Hari S. Sharma, Uppsala University, Sweden

Andrea Bozoki, Michigan State

University, USA

*Correspondence:

Xiaojuan Guo, College of Information Science and Technology, Beijing

Normal University, No. 19,

Xin JieKouWai St., HaiDian District,

Beijing, China

e-mail: gxj@bnu.edu.cn
Recent neuroimaging studies have revealed normal aging-related alterations in functional and structural brain networks such as the default mode network (DMN). However, less is understood about specific brain structural dependencies or interactions between brain regions within the DMN in the normal aging process. In this study, using Bayesian network (BN) modeling, we analyzed gray matter volume data from 109 young and 82 old subjects to characterize the influence of aging on associations between core brain regions within the DMN. Furthermore, we investigated the discriminability of the aging-associated BN models for the young and old groups. Compared to their young counterparts, the old subjects showed significant reductions in connections from right inferior temporal cortex (ITC) to medial prefrontal cortex (MPFC), right hippocampus (HP) to right ITC, and mPFC to posterior cingulate cortex and increases in connections from left HP to MPFC and right inferior parietal cortex to right ITC. Moreover, the classification results showed that the aging-related BN models could predict group membership with $88.48 \%$ accuracy, $88.07 \%$ sensitivity, and $89.02 \%$ specificity. Our findings suggest that structural associations within the DMN may be affected by normal aging and provide crucial information about aging effects on brain structural networks.

Keywords: normal aging, Bayesian network modeling, default mode network, structural associations, gray matter

\section{INTRODUCTION}

Normal aging is typically accompanied by progressive and gradual decline in memory and executive control functions together with morphological changes in the brain (Damoiseaux et al., 2008; Miller et al., 2008; Madden et al., 2010). A number of structural magnetic resonance imaging (MRI) studies have shown that normal aging-related morphological changes involve significant reductions in gray matter volume or cortical thickness (Good et al., 2001; Taki et al., 2004; Smith et al., 2007; Kalpouzos et al., 2009). Moreover, most of these studies have consistently depicted a common pattern of gray matter atrophy in the prefrontal cortex (Raz et al., 1997; Tisserand et al., 2002; Lemaitre et al., 2012) and the medial temporal lobe (Sullivan et al., 1995; Lemaitre et al., 2012). Interestingly, many important brain regions with high centrality (hubs) were located in the prefrontal regions, and these agingaffected regions were associated with deficits in cognitive functions (Bullmore and Sporns, 2009; Wu et al., 2012).

Most of the previous structural MRI studies focused on localizing brain regions using univariate statistical approaches, and they might have potentially missed the covariant morphometric information related to normal aging. Using multivariate analytical methods, several investigations have revealed the brain structure's small-world attributes [characterized by high degrees of local clustering among regions-of-interest (ROIs) and short paths linking all ROIs] or modularity (defined by distinct ROI groups with dense connections within each ROI-group and sparse connections between these ROI groups) (Bullmore and Sporns, 2009). These publications suggested that normal aging-related changes exhibited the organized inter-regional covariance of morphological features as a well-defined network (Bergfield et al., 2010; Chen et al., 2011; Montembeault et al., 2012). In addition, the scaled subprofile model (SSM) was also used to depict an age-related structural network, which showed concurrent decreases in gray matter volume, notably in the bilateral medial frontal, insula/perisylvian, and anterior cingulate regions (Bergfield et al., 2010). Finally, utilizing cortical thickness correlation analysis, Chen et al. (2011) found that aging was associated with organizational alterations of structural networks. Although these recent MRI studies have constructed structural networks associated with normal aging, less is known about the influence of aging on inter-region dependencies among spatially distributed regions within such brain networks.

On top of the above-mentioned structural networks, the human brain is also intrinsically organized into complicated functional networks (Bullmore and Sporns, 2009; Bassett and Gazzaniga, 2011; Sporns, 2011). It has been widely accepted that brain activity in resting state is organized into several functionally relevant networks (De Luca et al., 2006; Mantini et al., 2007) such as the default mode network (DMN), attention and 
visual/auditory networks. Literature findings suggest that these resting state functional networks are related to structural networks, therefore providing crucial insight into structural networks (or vice versa).

Among these functional networks, the DMN, with its core regions such as the posterior cingulate cortex (PCC) and medial prefrontal cortex (mPFC), is one of the most frequently discussed networks. For example, using a combination of diffusion tensor imaging (DTI) and resting state functional MRI, researchers have demonstrated that functional connectivity in resting state reflects structural connectivity within the DMN (Damoiseaux and Greicius, 2009; Greicius et al., 2009). Furthermore, normal aging was also related to alterations in functional connectivity in the DMN, especially decreased connectivity among the PCC, mPFC, and parietal cortex (Hafkemeijer et al., 2012). Additionally, using cortical thickness or gray matter volume, some researchers have indicated that old adults have distinctly reduced intra-module connections in the DMN when compared with young adults (Chen et al., 2011; Wu et al., 2012). Together, these findings suggested the needs for further exploring aging-associated alterations in the DMN in the context of the structural network organizational changes.

The organizational changes could be investigated using Bayesian network (BN) approach, which was introduced and utilized in neuroimaging studies (Zheng and Rajapakse, 2006). Without a prior model configuration, $\mathrm{BN}$ modeling can be used to investigate association dependency, or directed connection, of one ROI on another. Here, the directed connection is in the context of conditional probability (Chen and Herskovits, 2006; Zheng and Rajapakse, 2006). As a tool to investigate associations among variables, such as ROIs in neuroimaging studies, BN approach has been successfully applied to study functional networks in Alzheimer's disease (AD) (Wu et al., 2011) and structural networks in mild cognitive impairment (MCI) based on MRI (Chen and Herskovits, 2006; Chen et al., 2012). These findings indicated that $\mathrm{BN}$ approach was capable of characterizing associations among brain regions. The feasibility of using $\mathrm{BN}$ approach to investigate the effect of normal aging on structural networks, such as the $\mathrm{DMN}$, is however not well documented in the literature.

Using BN approach and structural MRI data from healthy young and old subjects, the current study aimed to explore the influence of aging on associations of regional gray matter volume among the core DMN regions. These structural associations are in terms of probabilistic dependence. The association differences between these two groups were assessed statistically by using a non-parametric permutation test. Finally, we investigated the discriminability of aging-associated BN models to classify young and old subjects.

\section{MATERIALS AND METHODS SUBJECTS}

All participants in this study were from the Open Access Series of Imaging Studies (OASIS) database ${ }^{1}$ including 109 young adults [22.73 \pm 2.34 years old (range: $20-28$ ), 65 females, and 44 males]

${ }^{1}$ http://www.oasis-brains.org and 82 healthy old adults [74.37 \pm 8.23 years old (range: $60-90$ ), 60 females, and 22 males]. Young subjects were recruited from the Washington University community and questioned about their medical histories and use of psychoactive drugs. Older adults were recruited from the Washington University's Alzheimer Disease Research Center (ADRC) and underwent ADRC's full assessment (Marcus et al., 2007). The dementia status of old adults was assessed by Mini-Mental State Examination (MMSE) (Folstein et al., 1975) and Clinical Dementia Rating (CDR) scores (Morris, 1993). In this study, the healthy old subjects (CDR $=0)$ had mean MMSE scores of $29.02 \pm 1.27$ (range: $25-30$ ). The young group did not differ from the old group in sex ratio $\left(\chi_{1}^{2}=3.792, p=0.051\right)$. All subjects participated in accordance with guidelines of the Washington University Human Studies Committee. The detailed demographics of all participants were described in Marcus et al. (2007) report.

\section{MRI ACQUISITION}

For each subject, three or four sagittally T1-weighted MPRAGE images were collected on a $1.5-\mathrm{T}$ MRI scanner $(\mathrm{TR} / \mathrm{TE} / \mathrm{TI}=$ 9.7/4.0/20 ms, flip angle $=10^{\circ}, \mathrm{FOV}=256 \mathrm{~mm} \times 256 \mathrm{~mm}$, voxel size $=1 \mathrm{~mm} \times 1 \mathrm{~mm}$, slices $=128$, thickness $=1.25 \mathrm{~mm})$. For the sake of increasing signal-to-noise ratio, the T1 image selected in this study for each subject was a motion-corrected coregistered average image $(1 \mathrm{~mm} \times 1 \mathrm{~mm} \times 1 \mathrm{~mm})$ of all available data (Marcus et al., 2007).

\section{IMAGE PRE-PROCESSING}

All of the structural T1 images were pre-processed using the VBM8 Toolbox ${ }^{2}$ in $\mathrm{SPM}^{3}{ }^{3}$. Using adaptive maximum posterior and partial volume estimation (Rajapakse et al., 1997; Tohka et al., 2004), the structural image for every subject was segmented into rigidbody aligned gray matter, white matter, and cerebrospinal fluid (CSF) maps. Two denoising methods, the spatially adaptive nonlocal means denoising filter and classical Markov Random Field approach, were applied to improve the image segmentation. The gray matter image was normalized to the Montreal Neurological Institute (MNI) space by high dimensional diffeomorphic anatomical registration using exponential Lie algebra (DARTEL) algorithm (Ashburner, 2007). DARTEL parameterizes diffeomorphic and inverse consistent deformations using a time-invariant velocity field. The normalized gray matter maps were modulated by Jacobian determinants from the deformations to preserve the total amount of tissue in the native spaces. Finally, all of the gray matter maps were smoothed with a Gaussian kernel of $8 \mathrm{~mm}$ full width at half maximum (FWHM).

\section{ROIs DEFINITION}

We selected eight core ROIs based on previous studies (Fox et al., 2005; Fair et al., 2008). Table 1 shows the names and corresponding abbreviations of these eight ROIs. Each ROI mask was generated by using the WFU_PickAtlas software ${ }^{4}$ (Maldjian et al., 2003). Every ROI covered the entire area of the corresponding anatomical

\footnotetext{
${ }^{2}$ http://dbm.neuro.uni-jena.de/vbm8

${ }^{3}$ http://www.fil.ion.ucl.ac.uk/spm

${ }^{4} \mathrm{http}: / /$ www.ansir.wfubmc.edu
} 
Table 1 | Brain regions and the corresponding abbreviations of eight ROIs in the DMN.

\section{Brain regions}

Abbreviations

Posterior cingulate cortex

Medial prefrontal cortex

Left hippocampus

Right hippocampus

Left inferior parietal cortex

Right inferior parietal cortex

Left inferior temporal cortex

Right inferior temporal cortex parameter of node $j$. All of the procedures were implemented with the Bayesian Net Toolbox ${ }^{5}$ in MATLAB R2010.

After constructing the BN model, the conditional probability density can be calculated for node $j$ given its parent node-set $\pi_{j}$, which directly connects with node $j$ in the graph structure:

$$
\begin{aligned}
& p\left(X_{j} \mid \pi_{j}, \theta_{j}\right) \\
& \quad=\frac{1}{(2 \pi)^{1 / 2}|\Sigma|^{1 / 2}} \exp \left\{-\frac{1}{2}\left(X_{j}-\mu_{j}\right)^{T} \Sigma^{-1}\left(X_{j}-\mu_{j}\right)\right\}
\end{aligned}
$$

where $\mu_{j}$ and $\Sigma_{j}$ respectively represent the conditional mean and variance of $X_{j} ; \theta_{j}$ is the parameter for node $j$. Then, the joint probability density for all of the ROIs can be described as $p(X)=\prod_{j=1}^{d} p\left(X_{j} \mid \pi_{j}, \theta_{j}\right)$.

A non-parametric permutation test with 5000 permutations on the distributions of all subjects was employed to detect the differences in all connection weight coefficients between two BN models. Here, the connection is about the existence of an edge between two brain regions in the $\mathrm{BN}$ models and represents a statistical association between the corresponding variables. And the weight coefficient represents the strength of structural association or the volumetric correlation strength. In the end, we assessed the significance of the difference by calculating the type-I error probability of Young $>$ Old or Old $>$ Young.

Additionally, we used the joint probability density values to assign the membership of a subject to one of the two groups and used the receiver operating curve (ROC) to assess the discriminability.

\section{RESULTS}

\section{STRUCTURAL ASSOCIATIONS WITHIN THE DMN}

Figure 1 shows two BN models for the young and old groups, representing structural associations or probabilistic dependence among the core DMN regions. Table 2 lists the corresponding connection directions and weight coefficients. Connections $\mathrm{lHP} \rightarrow \mathrm{mPFC}, \mathrm{rITC} \rightarrow \mathrm{lITC}$, and $\mathrm{rITC} \rightarrow \mathrm{mPFC}$ were present in both young and old groups, connections $\mathrm{rHP} \rightarrow \mathrm{rITC}, \mathrm{PCC} \rightarrow \mathrm{IIPC}, \mathrm{mPFC} \rightarrow \mathrm{PCC}$, and $\mathrm{mPFC} \rightarrow \mathrm{IIPC}$ were observed only in the young group, and connections $\mathrm{IITC} \rightarrow \mathrm{lHP}, \mathrm{rIPC} \rightarrow$ rITC, and PCC $\rightarrow$ rIPC were observed only in the old group. Although connections between rHP and IHP, rIPC and IIPC were revealed in both groups, they were opposite in direction.

\section{BETWEEN-GROUP ASSOCIATION DIFFERENCES}

We adopted a permutation test to investigate between-group differences in the connection weight coefficients with the probabilities of type-I errors listed in Table 3. The column "Young $>$ Old" shows the probabilities of type-I errors under the null hypothesis that the strength of structural association in the young group is stronger than in the old group, but the column "Old > Young" displays the opposite. The connections $\mathrm{rITC} \rightarrow \mathrm{mPFC}, \mathrm{rHP} \rightarrow \mathrm{rITC}$, and $\mathrm{mPFC} \rightarrow$ PCC were stronger in the young group than in the

${ }^{5}$ https://code.google.com/p/bnt/ 


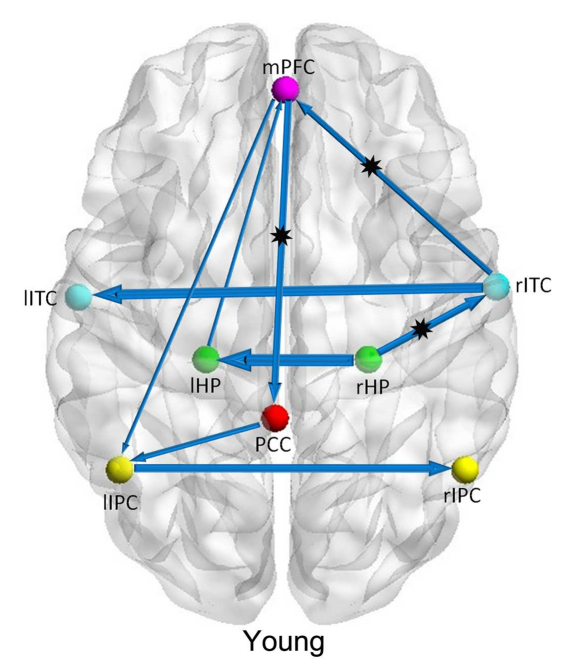

FIGURE 1 | Bayesian network models of the DMN in the young (left panel) and old (right panel) groups. The arrows represent dependencies among brain regions and the thickness of the arrows is

Table 2 | List of connections and the corresponding weight coefficients in the Bayesian network models of the young and old groups.

\begin{tabular}{|c|c|c|c|}
\hline \multicolumn{2}{|c|}{ Connections } & \multicolumn{2}{|c|}{$\begin{array}{l}\text { Weight coefficients } \\
\text { of connections }\end{array}$} \\
\hline & & Young & Old \\
\hline \multirow[t]{3}{*}{ 1 } & $\mathrm{IHP} \rightarrow \mathrm{mPFC}$ & 0.284 & 0.292 \\
\hline & $\mathrm{rlTC} \rightarrow \mathrm{IITC}$ & 0.784 & 0.723 \\
\hline & $\mathrm{rITC} \rightarrow \mathrm{mPFC}$ & 0.469 & 0.372 \\
\hline \multirow[t]{4}{*}{ ॥ } & $\mathrm{rHP} \rightarrow \mathrm{rlTC}$ & 0.576 & \\
\hline & $\mathrm{PCC} \rightarrow \| \mathrm{IPC}$ & 0.422 & \\
\hline & $\mathrm{mPFC} \rightarrow \mathrm{PCC}$ & 0.548 & \\
\hline & $\mathrm{mPFC} \rightarrow \| \mathrm{IPC}$ & 0.292 & \\
\hline \multirow[t]{3}{*}{ III } & $\mathrm{IITC} \rightarrow \mathrm{IHP}$ & & 0.425 \\
\hline & $\mathrm{rlPC} \rightarrow \mathrm{rlTC}$ & & 0.559 \\
\hline & $\mathrm{PCC} \rightarrow \mathrm{rlPC}$ & & 0.576 \\
\hline \multirow[t]{4}{*}{ IV } & $\mathrm{rHP} \rightarrow \mathrm{IHP}$ & 0.921 & \\
\hline & $\mathrm{IHP} \rightarrow \mathrm{rHP}$ & & 0.773 \\
\hline & $\mathrm{rIPC} \rightarrow \| \mathrm{IPC}$ & & 0.649 \\
\hline & $\| \mathrm{PC} \rightarrow \mathrm{rIPC}$ & 0.625 & \\
\hline
\end{tabular}

Part I of this table lists connections that exist in both groups. Parts II and III show the connections present only in the young and old groups, respectively. Part IV lists connections that are opposite in direction in two groups.

old group, which were determined by a significance level of uncorrected $p<0.05$. Using the same assessment, the $\mathrm{lHP} \rightarrow \mathrm{mPFC}$ and $\mathrm{rIPC} \rightarrow$ rITC connections showed significantly decreased alterations in the young group when compared with the old group.

\section{CLASSIFICATION ABILITY}

The classification results for the young versus the old groups are summarized as follows. The classification accuracy based on the

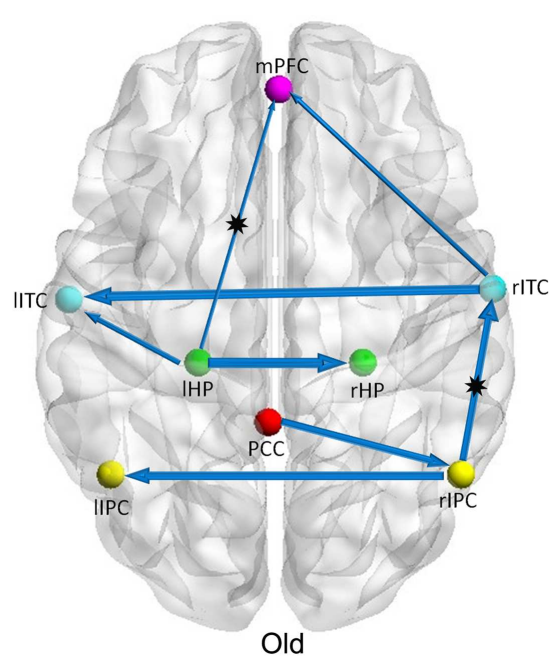

proportional to the strength of the connections. The asterisks indicate the connections that were significantly stronger in young/old than in old/young groups.

Table 3 |The probabilities of Type-I errors for between-group differences in all connections.

\begin{tabular}{|c|c|c|c|}
\hline \multicolumn{2}{|c|}{ Young $>$ Old } & \multicolumn{2}{|c|}{ Old $>$ Young } \\
\hline Connections & Probabilities & Connections & Probabilities \\
\hline $\mathrm{IHP} \rightarrow \mathrm{mPFC}$ & 1.000 & $\mathrm{IHP} \rightarrow \mathrm{mPFC}$ & 0.000 \\
\hline $\mathrm{rITC} \rightarrow \| \mathrm{ITC}$ & 0.334 & $\mathrm{rITC} \rightarrow \mathrm{IITC}$ & 0.666 \\
\hline $\mathrm{rlTC} \rightarrow \mathrm{mPFC}$ & 0.000 & $\mathrm{rlTC} \rightarrow \mathrm{mPFC}$ & 1.000 \\
\hline $\mathrm{rHP} \rightarrow \mathrm{rITC}$ & 0.041 & $\| \mathrm{TC} \rightarrow \mathrm{IHP}$ & 0.064 \\
\hline $\mathrm{PCC} \rightarrow \| \mathrm{IPC}$ & 0.150 & $\mathrm{rIPC} \rightarrow \mathrm{rITC}$ & 0.000 \\
\hline $\mathrm{mPFC} \rightarrow \mathrm{PCC}$ & 0.050 & $\mathrm{PCC} \rightarrow \mathrm{rIPC}$ & 0.832 \\
\hline $\mathrm{mPFC} \rightarrow \| \mathrm{IPC}$ & 0.220 & $\mathrm{IHP} \rightarrow \mathrm{rHP}$ & 0.145 \\
\hline $\mathrm{rHP} \rightarrow \mathrm{IHP}$ & 0.232 & $\mathrm{rlPC} \rightarrow \| \mathrm{IPC}$ & 0.236 \\
\hline$\| \mathrm{PC} \rightarrow \mathrm{rIPC}$ & 0.233 & & \\
\hline
\end{tabular}

The column "Young > Old" displays the probabilities of type-l errors in the hypothesis that the strength of connections in the young group is stronger than in the old group. The other column "Old > Young" shows the opposite. The probabilities marked in bold indicate significantly stronger connections $(p<0.05)$.

derived two $\mathrm{BN}$ models reached $88.48 \%$, and the corresponding specificity and sensitivity were 89.02 and $88.07 \%$, respectively.

\section{DISCUSSION}

In the present study, we applied BN method to characterize gray matter associations among core brain regions within the DMN of young and old adults. Then, we employed a non-parametric permutation test to detect the BN connection differences in weight coefficients between two groups. Furthermore, we evaluated the discriminability of the aging-related $\mathrm{BN}$ models by comparing joint probability density scores based on the BN models in each of the young and old groups. The permutation test showed significant reductions in the connections $\mathrm{rITC} \rightarrow \mathrm{mPFC}, \mathrm{rHP} \rightarrow \mathrm{rITC}$, 
and $\mathrm{mPFC} \rightarrow$ PCC and increases in the connections $\mathrm{lHP} \rightarrow \mathrm{mPFC}$ and $\mathrm{rIPC} \rightarrow$ IITC in the old group when compared with the young group. In addition, the aging-related $\mathrm{BN}$ models could predict the membership of subjects with high accuracy, sensitivity, and specificity.

In contrast to the BN model in young adults, the one in old adults revealed some coordination disruptions among the DMN core regions, possibly due to aging. Our findings, in this regard, are consistent with several published studies. For example, using correlation analysis, previous studies based on cortical thickness or gray matter volume consistently demonstrated that intra-modular connections in the DMN in the old group were more reduced than in the young group (Chen et al., 2011; Wu et al., 2012). Additionally, Hafkemeijer et al. (2012) summarized various agerelated studies of brain function and found that the DMN generally showed reduced functional connectivity as a consequence of the normal aging process. Decreased functional connectivity might reflect structural alteration of brain network.

Our findings revealed that there were strong structural associations between brain regions in one hemisphere and the homologous regions in the opposite hemisphere in both young and old groups. However, the strengths of structural associations were generally greater in the young group than in the old group. The decreased strengths of structural associations between two homologous brain regions reflected inconsistency in the degree of atrophy between right and left hemispheres in the old group. Some previous studies attempted to explore the possible causes for the reduced homologous inter-hemisphere connections. For example, Vernooij et al. (2008) found white matter atrophy in corpus callosum might lead to a decrease in structural connections between bilateral brain regions. In another study, Mechelli et al. (2005) proposed that the gray matter density of a brain region could predict the density of a homologous region located in the opposite hemisphere. In addition to the fact that corpus callosum contributed to the inter-hemispheric structural connectivity, a previous study demonstrated that loss of corpus callosum integrity affected functional connectivity (Quigley et al., 2003).

The prominent between-group changes in the strengths of structural associations included the decreased connections from rITC to mPFC, rHP to rITC, and mPFC to PCC and increased connections from IHP to mPFC and rIPC to rITC in the old group compared with the young group. The prefrontal cortex is well known to be associated with executive control function (Madden et al., 2010). Overall, in our study, the number of connections with $\mathrm{mPFC}$ in the old group was reduced when compared with the young group. In a previous DTI study of normal aging, Grieve et al. (2007) found that prefrontal regions showed notable negative relationship with age, which could be a factor that affected the strengths of structural associations between other regions and mPFC as proposed in our study. Another study, based on seedROI, verified that the functionally correlated DMN regions were also volumetrically correlated and that such correlations between the right angular cortex and some frontal regions were significantly decreased in the old group (Montembeault et al., 2012). This observed alteration is consistent with our present result showing connections between MPFC and IIPC present only in the young group. Moreover, Vernooij et al. (2008) found that fractional anisotropy in cingulate bundle was reduced, which might result in decreased structural associations between mPFC and PCC found in the current study. Andrews-Hanna et al. (2007) also reported that older adults showed decreased functional connectivity between mPFC and PCC. Additionally, using cortical thickness, Chen et al. (2011) showed that the correlation between right $\mathrm{mPFC}$ and left precuneus was decreased due to aging, which is also consistent with our findings. Pertaining to increased structural associations in the old group, it was possible that these results were due to a connectivity compensation, a concept described as some brain regions working harder to make up for the deficiencies of other regions in the network (Cappell, 2008).

Additionally, we noted that a small number of connections (between IHP and rHP, between IIPC and rIPC) had reversal direction in the two groups but showed no statistically significant between-group differences. We speculated that the direction alternation might be influenced by aging among other factors. The explanation on the direction reversal should be with great caution since the association dependency is in terms of the conditional probability (Chen and Herskovits, 2006). More importantly, Smith et al. (2011) suggested that it was more difficult to achieve accurate estimation of connection directionality by $\mathrm{BN}$ approach in spite of its high sensitivity of detecting the presence of connections.

Bayesian network modeling can be used to examine probabilistic associations among variables. Till now, $\mathrm{BN}$ approach has been successfully utilized in neuroimaging (including functional MRI and structural MRI) studies (Chen and Herskovits, 2006; Zheng and Rajapakse, 2006; Wu et al., 2011; Chen et al., 2012). For functional MRI data, $\mathrm{BN}$ modeling examines conditional dependencies of brain activity based on functional MRI time series for each individual subject. For structural MRI data, BN modeling investigates probabilistic associations of morphological feature based on morphometric variables such as gray matter volume from all subjects at the group level either within or between groups. A number of publications have suggested that brain regions covary in their morphological properties, and such structural networks coordinate due to various factors such as normal aging (Bergfield et al., 2010; Chen et al., 2011; Montembeault et al., 2012). Furthermore, some previous studies proposed that structural covariances may result from mutually trophic influences or common experience-related plasticity that are mediated by white matter connections (Ferrer et al., 1995; Mechelli et al., 2005; Soriano-Mas et al., 2013) and the altered relation between regions may arise from lack of mutually trophic influences in different clinical conditions (He et al., 2008; Seeley et al., 2009). Therefore, BN modeling, as a valuable method of mining association relationships between continuous variables, can be used to investigate the association dependency based on regional gray matter volumes. Nevertheless, our current results on the directional relationship are statistical in nature and they cannot replace direct biological and medical evidence. We hope that our study provides additional, consistent but preliminary findings in support of more comprehensive investigations in this regard.

In addition to examining the network differences, we employed the $\mathrm{BN}$ model as a classification tool to infer group membership of subjects by comparing the joint probability densities between the young and the old groups. This operation for classification integrated all gray matter volume information from eight ROIs 
instead of focusing on obvious morphological changes in some particular brain regions. The ROC analysis demonstrated the discriminability of the $\mathrm{BN}$ model with $88.07 \%$ sensitivity, $89.02 \%$ specificity, and $88.48 \%$ accuracy. Both the sensitivity and specificity were close to $90 \%$, which not only verified the validity of our age-associated $\mathrm{BN}$ models but also provided an evidence for the $\mathrm{BN}$ model to server as a predictive brain biomarker in structure for normal aging.

Although the accuracy of classification was close to $90 \%$, we noted that we examined the discriminability of the derived $\mathrm{BN}$ models by comparing joint probability densities of the subjects used to construct BN models. This classification of post hoc nature has limited validity. Thus, its generalizability needs to be crossvalidated using independent dataset. Additional studies are needed to verify the replicability and stability of the aging-related $\mathrm{BN}$ models in an independent dataset.

In summary, our study suggests that structural associations within the DMN are affected by the normal aging process. The $\mathrm{BN}$ modeling approach potentially can serve as a useful tool for studying structural associations or probabilistic dependence among multiple brain regions.

\section{ACKNOWLEDGMENTS}

This work was supported by the National Key Basic Research Program (973 Program), China (2012CB720704), the National Hightech R\&D Program (863 Program), China (2012AA011603), the National Natural Science Foundation (NNSF), China (81000603), Key Program of NNSF, China (60931003), the Funds for International Cooperation and Exchange of NNSF, China (61210001), the Fundamental Research Funds for the Central Universities of China, the National Institute of Mental Health, US (RO1 MH57899), the National Institute on Aging, US (9R01AG03158110, P30 AG19610, k23 AG24062), and the State of Arizona. In addition, we used the data from Open Access Series of Imaging Studies (OASIS). OASIS project was funded by the following grants: P50 AG05681, P01 AG03991, R01 AG021910, P50 MH071616, U24 RR021382, and R01 MH56584.

\section{REFERENCES}

Andrews-Hanna, J. R., Snyder, A. Z., Vincent, J. L., Lustig, C., Head, D., Raichle, M. E., et al. (2007). Disruption of large-scale brain systems in advanced aging. Neuron 56, 924-935. doi:10.1016/j.neuron.2007.10.038

Ashburner, J. (2007). A fast diffeomorphic image registration algorithm. Neuroimage 38, 95-113. doi:10.1016/j.neuroimage.2007.07.007

Bassett, D. S., and Gazzaniga, M. S. (2011). Understanding complexity in the human brain. Trends Cogn. Sci. 15, 200-209. doi:10.1016/j.tics.2011.03.006

Bergfield, K. L., Hanson, K. D., Chen, K., Teipel, S. J., Hampel, H., Rapoport, S. I., et al. (2010). Age-related networks of regional covariance in MRI gray matter: reproducible multivariate patterns in healthy aging. Neuroimage 49, 1750-1759. doi:10.1016/j.neuroimage.2009.09.051

Bullmore, E., and Sporns, O. (2009). Complex brain networks: graph theoretical analysis of structural and functional systems. Nat. Rev. Neurosci. 10, 186-198. doi:10.1038/nrn2575

Cappell, P. A. R. K. (2008). Neurocognitive aging and the compensation hypothesis. Curr. Dir. Psychol. Sci. 17, 177-182. doi:10.1111/j.1467-8721.2008.00570.x

Chen, R., and Herskovits, E. H. (2006). Network analysis of mild cognitive impairment. Neuroimage 29, 1252-1259. doi:10.1016/j.neuroimage.2005.08.020

Chen, R., Resnick, S. M., Davatzikos, C., and Herskovits, E. H. (2012). Dynamic Bayesian network modeling for longitudinal brain morphometry. Neuroimage 59, 2330-2338. doi:10.1016/j.neuroimage.2011.09.023
Chen, Z. J., He, Y., Rosa-Neto, P., Gong, G., and Evans, A. C. (2011). Age-related alterations in the modular organization of structural cortical network by using cortical thickness from MRI. Neuroimage 56, 235-245. doi:10.1016/j.neuroimage. 2011.01.010

Chickering, D. M. (2003). Optimal structure identification with greedy search. J. Mach. Learn. Res. 3, 507-554. doi:10.1016/j.neunet.2013.01.025

Damoiseaux, J. S., Beckmann, C. F., Arigita, E. J., Barkhof, F., Scheltens, P., Stam, C. J., et al. (2008). Reduced resting-state brain activity in the "default network" in normal aging. Cereb. Cortex 18, 1856-1864. doi:10.1093/cercor/bhm207

Damoiseaux, J. S., and Greicius, M. D. (2009). Greater than the sum of its parts: a review of studies combining structural connectivity and resting-state functional connectivity. Brain Struct. Funct. 213, 525-533. doi:10.1007/s00429009-0208-6

De Luca, M., Beckmann, C. F., De Stefano, N., Matthews, P. M., and Smith, S. M. (2006). fMRI resting state networks define distinct modes of long-distance interactions in the human brain. Neuroimage 29, 1359-1367. doi:10.1016/j. neuroimage.2005.08.035

Fair, D. A., Cohen, A. L., Dosenbach, N. U., Church, J. A., Miezin, F. M., Barch, D. M., et al. (2008). The maturing architecture of the brain's default network. Proc. Natl. Acad. Sci. U.S.A. 105, 4028-4032. doi:10.1073/pnas.0800376105

Ferrer, I., Blanco, R., Carulla, M., Condom, M., Alcantara, S., Olive, M., et al. (1995). Transforming growth factor-alpha immunoreactivity in the developing and adult brain. Neuroscience 66, 189-199. doi:10.1016/0306-4522(94)00584-R

Folstein, M. F., Folstein, S. E., and Mchugh, P. R. (1975). "Mini-mental state". A practical method for grading the cognitive state of patients for the clinician. J. Psychiatr. Res. 12, 189-198. doi:10.1016/0022-3956(75)90026-6

Fox, M. D., Snyder, A. Z., Vincent, J. L., Corbetta, M., Van Essen, D. C., and Raichle, M. E. (2005). The human brain is intrinsically organized into dynamic, anticorrelated functional networks. Proc. Natl. Acad. Sci. U.S.A. 102, 9673-9678. doi:10.1073/pnas.0504136102

Good, C. D., Johnsrude, I. S., Ashburner, J., Henson, R. N., Friston, K. J., and Frackowiak, R. S. (2001). A voxel-based morphometric study of ageing in 465 normal adult human brains. Neuroimage 14,21-36. doi:10.1006/nimg.2001.0857

Greicius, M. D., Supekar, K., Menon, V., and Dougherty, R. F. (2009). Resting-state functional connectivity reflects structural connectivity in the default mode network. Cereb. Cortex 19, 72-78. doi:10.1093/cercor/bhn059

Grieve, S. M., Williams, L. M., Paul, R. H., Clark, C. R., and Gordon, E. (2007). Cognitive aging, executive function, and fractional anisotropy: a diffusion tensor $\mathrm{MR}$ imaging study. Am. J. Neuroradiol. 28, 226-235.

Hafkemeijer, A., van der Grond, J., and Rombouts, S. A. (2012). Imaging the default mode network in aging and dementia. Biochim. Biophys. Acta 1822, 431-441. doi:10.1016/j.bbadis.2011.07.008

He, Y., Chen, Z., and Evans, A. (2008). Structural insights into aberrant topological patterns of large-scale cortical networks in Alzheimer's disease. J. Neurosci. 28, 4756-4766. doi:10.1523/JNEUROSCI.0141-08.2008

Kalpouzos, G., Chetelat, G., Baron, J. C., Landeau, B., Mevel, K., Godeau, C., et al. (2009). Voxel-based mapping of brain gray matter volume and glucose metabolism profiles in normal aging. Neurobiol. Aging 30, 112-124. doi:10.1016/j.neurobiolaging.2007.05.019

Lemaitre, H., Goldman, A. L., Sambataro, F., Verchinski, B. A., Meyer-Lindenberg, A., Weinberger, D. R., et al. (2012). Normal age-related brain morphometric changes: nonuniformity across cortical thickness, surface area and gray matter volume? Neurobiol. Aging 33, 611-617. doi:10.1016/j.neurobiolaging. 2010.07.013

Madden, D. J., Costello, M. C., Dennis, N. A., Davis, S. W., Shepler, A. M., Spaniol, J., et al. (2010). Adult age differences in functional connectivity during executive control. Neuroimage 52, 643-657. doi:10.1016/j.neuroimage.2010.04.249

Maldjian, J. A., Laurienti, P. J., Kraft, R. A., and Burdette, J. H. (2003). An automated method for neuroanatomic and cytoarchitectonic atlas-based interrogation of fMRI data sets. Neuroimage 19, 1233-1239. doi:10.1016/S10538119(03)00169-1

Mantini, D., Perrucci, M. G., Del, G. C., Romani, G. L., and Corbetta, M. (2007). Electrophysiological signatures of resting state networks in the human brain. Proc. Natl. Acad. Sci. U.S.A. 104, 13170-13175. doi:10.1073/pnas. 0700668104

Marcus, D. S., Wang, T. H., Parker, J., Csernansky, J. G., Morris, J. C., and Buckner, R. L. (2007). Open Access Series of Imaging Studies (OASIS): cross-sectional MRI data in young, middle aged, nondemented, and demented older adults. J. Cogn. Neurosci. 19, 1498-1507. doi:10.1162/jocn.2007.19.9.1498 
Mechelli, A., Friston, K. J., Frackowiak, R. S., and Price, C. J. (2005). Structural covariance in the human cortex. J. Neurosci. 25, 8303-8310. doi:10.1523/JNEUROSCI. 0357-05.2005

Miller, S. L., Celone, K., Depeau, K., Diamond, E., Dickerson, B. C., Rentz, D., et al. (2008). Age-related memory impairment associated with loss of parietal deactivation but preserved hippocampal activation. Proc. Natl. Acad. Sci. U.S.A. 105, 2181-2186. doi:10.1073/pnas.0706818105

Montembeault, M., Joubert, S., Doyon, J., Carrier, J., Gagnon, J. F., Monchi, O., et al. (2012). The impact of aging on gray matter structural covariance networks. Neuroimage 63, 754-759. doi:10.1016/j.neuroimage.2012.06.052

Morris, J. C. (1993). The Clinical Dementia Rating (CDR): current version and scoring rules. Neurology 43, 2412-2414. doi:10.1212/WNL.43.11.2412-a

Quigley, M., Cordes, D., Turski, P., Moritz, C., Haughton, V., Seth, R., et al. (2003). Role of the corpus callosum in functional connectivity. Am. J. Neuroradiol. 24, 208-212.

Rajapakse, J. C., Giedd, J. N., and Rapoport, J. L. (1997). Statistical approach to segmentation of single-channel cerebral MR images. IEEE Trans. Med. Imaging 16, 176-186. doi:10.1109/42.563663

Raz, N., Gunning, F. M., Head, D., Dupuis, J. H., Mcquain, J., Briggs, S. D., et al. (1997). Selective aging of the human cerebral cortex observed in vivo: differential vulnerability of the prefrontal gray matter. Cereb. Cortex 7, 268-282. doi:10.1093/cercor/7.3.268

Seeley, W. W., Crawford, R. K., Zhou, J., Miller, B. L., and Greicius, M. D. (2009). Neurodegenerative diseases target large-scale human brain networks. Neuron 62, 42-52. doi:10.1016/j.neuron.2009.03.024

Smith, C. D., Chebrolu, H., Wekstein, D. R., Schmitt, F. A., and Markesbery, W. R. (2007). Age and gender effects on human brain anatomy: a voxelbased morphometric study in healthy elderly. Neurobiol. Aging 28, 1075-1087. doi:10.1016/j.neurobiolaging.2006.05.018

Smith, S. M., Miller, K. L., Salimi-Khorshidi, G., Webster, M., Beckmann, C. F., Nichols, T. E., et al. (2011). Network modelling methods for FMRI. Neuroimage 54, 875-891. doi:10.1016/j.neuroimage.2010.08.063

Soriano-Mas, C., Harrison, B. J., Pujol, J., Lopez-Sola, M., Hernandez-Ribas, R., Alonso, P., et al. (2013). Structural covariance of the neostriatum with regional gray matter volumes. Brain Struct. Funct. 218, 697-709. doi:10.1007/s00429012-0422-5

Sporns, O. (2011). The human connectome: a complex network. Ann. N. Y. Acad. Sci. 1224, 109-125. doi:10.1111/j.1749-6632.2010.05888.x

Sullivan, E. V., Marsh, L., Mathalon, D. H., Lim, K. O., and Pfefferbaum, A. (1995). Age-related decline in MRI volumes of temporal lobe gray matter but not hippocampus. Neurobiol. Aging 16, 591-606. doi:10.1016/0197-4580(95)00074-O
Taki, Y., Goto, R., Evans, A., Zijdenbos, A., Neelin, P., Lerch, J., et al. (2004). Voxelbased morphometry of human brain with age and cerebrovascular risk factors. Neurobiol. Aging 25, 455-463. doi:10.1016/j.neurobiolaging.2003.09.002

Tisserand, D. J., Pruessner, J. C., Sanz, A. E., van Boxtel, M. P., Evans, A. C., Jolles, J., et al. (2002). Regional frontal cortical volumes decrease differentially in aging: an MRI study to compare volumetric approaches and voxel-based morphometry. Neuroimage 17, 657-669. doi:10.1006/nimg.2002.1173

Tohka, J., Zijdenbos, A., and Evans, A. (2004). Fast and robust parameter estimation for statistical partial volume models in brain MRI. Neuroimage 23, 84-97. doi:10.1016/j.neuroimage.2004.05.007

Vernooij, M. W., de Groot, M., van der Lugt, A., Ikram, M. A., Krestin, G. P., Hofman, A., et al. (2008). White matter atrophy and lesion formation explain the loss of structural integrity of white matter in aging. Neuroimage 43, 470-477. doi:10.1016/j.neuroimage.2008.07.052

Wu, K., Taki, Y., Sato, K., Kinomura, S., Goto, R., Okada, K., et al. (2012). Age-related changes in topological organization of structural brain networks in healthy individuals. Hum. Brain Mapp. 33, 552-568. doi:10.1002/hbm.21232

Wu, X., Li, R., Fleisher, A. S., Reiman, E. M., Guan, X., Zhang, Y., et al. (2011). Altered default mode network connectivity in Alzheimer's disease - a resting functional MRI and Bayesian network study. Hum. Brain Mapp. 32, 1868-1881. doi:10.1002/hbm.21153

Zheng, X., and Rajapakse, J. C. (2006). Learning functional structure from fMR images. Neuroimage 31, 1601-1613. doi:10.1016/j.neuroimage.2006.01.031

Conflict of Interest Statement: The authors declare that the research was conducted in the absence of any commercial or financial relationships that could be construed as a potential conflict of interest.

Received: 08 October 2013; paper pending published: 15 February 2014; accepted: 14 May 2014; published online: 30 May 2014.

Citation: Wang Y, Chen K, Zhang J, Yao L, Li K, Jin Z, Ye Q and Guo X (2014) Aging influence on gray matter structural associations within the default mode network utilizing Bayesian network modeling. Front. Aging Neurosci. 6:105. doi: 10.3389/fnagi.2014.00105

This article was submitted to the journal Frontiers in Aging Neuroscience. Copyright (C) 2014 Wang, Chen, Zhang, Yao, Li, Jin, Ye and Guo. This is an openaccess article distributed under the terms of the Creative Commons Attribution License (CC BY). The use, distribution or reproduction in other forums is permitted, provided the original author(s) or licensor are credited and that the original publication in this journal is cited, in accordance with accepted academic practice. No use, distribution or reproduction is permitted which does not comply with these terms. 